\title{
Finite frequency model reduction for 2-D fuzzy systems in FM model
}

\author{
Rachid Naoual ${ }^{1, *}$, Abderrahim El-Amrani ${ }^{2, * *}$, and Ismail BOUMHIDI $^{2, * * *}$ \\ ${ }^{1}$ Department of Engineering, Higher Institute of Engineering and Business (ISGA), Fez, Morroco. \\ ${ }^{2}$ LISAC Laboratory, FSDM, Sidi Mohamed Ben Abdellah University, Fes, Morocco.
}

\begin{abstract}
This paper deals with the problem of $H_{\infty}$ model reduction for two-dimensional (2D) discrete TakagiSugeno (T-S) fuzzy systems described by Fornasini-Marchesini local state-space (FM LSS) models, over finite frequency (FF) domain. New design conditions guaranteeing the FF $H_{\infty}$ model reduction are established in terms of Linear Matrix Inequalities (LMIs). To highlight the effectiveness of the proposed $H_{\infty}$ model reduction design, a numerical example is given.
\end{abstract}

\section{Introduction}

During the past decades, much progress has been made for 2D systems in the literature [1,2], many important results based on LMI approach have already been reported. Among these results, stability analysis and stabilization design for 2D systems have been studied in [3], $H_{\infty}$ filtering problem can be found in [14], model reduction problem in [6].

The $H_{\infty}$ model reduction aims to find a low-order model for a given model such that the $H_{\infty}$ norm of the error between these two models is minimized or satisfies the specific performance. Many results on $H_{\infty}$ model reduction have been reported in the literature [6,9-11].

The point of interest in aforementioned literature is that all performance indices are defined in the entire frequency (EF) domain. However, in major real applications, the design characteristics are usually given in specific frequency domains. So, the standard design approaches for the whole frequency domain may bring conservatism $[15,18],[17][12,13,16]$.

In this paper, we consider the $H_{\infty}$ model reduction for 2D T-S fuzzy Fornasini-Marchesini Models. Sufficient conditions for the existence of solutions are parameterized in LMI form. An explicit parameterization of the desired reduced-order models is given. Finally, a numerical example is provided to prove the effectiveness of FF propose method.

Notations Superscript " $T$ " stands for matrix transposition. In symmetric block matrices or long matrix expressions, we use an asterisk " $*$ " to represent a term that is induced by symmetry. Notation $P>0$ means that matrix $P$ is positive. $I$ denotes an identity matrix with appropriate

\footnotetext{
*e-mail: rachid.naoual@isga.ma

**e-mail: abderrahim.elamrani@usmba.ac.ma

***e-mail: ismail.boumhidi@usmba.ac.ma
}

dimension. Generally, $\operatorname{sym}\{A\}$ denotes $A+A^{T}, \operatorname{diag}\{.$. stands for block diagonal matrix. The $l_{2}$ norm for a $2 \mathrm{D}$ signal $u(i, j)$ is given by

$$
\|u\|_{2}=\sqrt{\sum_{i=0}^{\infty} \sum_{j=0}^{\infty} u^{T}(i, j) u(i, j)}
$$

where $u(i, j)$ is said to be in the space $l_{2}\{[0, \infty),[0, \infty)\}$ or $l_{2}$, for simplicity, if $\|u\|_{2}<\infty$. A 2D signal $u(i, j)$ in the $l_{2}$ space is an energy-bounded signal.

\section{Problem description and preliminaries}

\subsection{Problem description}

In this paper, we consider a class of 2D nonlinear discretetime systems described by the following T-S FMLSS fuzzy model

Plant Rule I: IF $\theta_{1}(k)$ is $\tilde{N}_{1}^{l}, \theta_{2}(k)$ is $\tilde{N}_{2}^{l}, \ldots$ and $\theta_{\alpha}(k)$ is $\tilde{N}_{\alpha}^{l}$, Then,

$$
\begin{aligned}
x_{i+1, j+1} & =A_{1 l} x_{i, j+1}+A_{2 l} x_{i+1, j}+B_{1 l} u_{i, j+1}+B_{2 l} u_{i+1, j} \\
y_{i, j} & =C_{l} x_{i, j}
\end{aligned}
$$

where $\left(\tilde{N}_{1}^{l}, \ldots, \tilde{N}_{\alpha}^{l}\right)$ are the fuzzy sets; $l$ is the number of IFTHEN rules $(l=1,2, \ldots, r) ; \theta(k)=\left[\theta_{1}(k), \theta_{2}(k), \ldots, \theta_{\alpha}(k)\right]$ are the premise variables; $k=\{(i, j+1),(i+1, j)\}$; $x(i, j) \in \mathbb{R}^{n}$ is the state vector; $y(i, j) \in \mathbb{R}^{n_{y}}$ is the measured output; $u(i, j) \in \mathbb{R}^{p}$ is the noise input (that belongs to $\left.l_{2}\{[0, \infty),[0, \infty)\}\right) ;\left(A_{1 l}, B_{1 l}, A_{2 l}, B_{2 l}, C_{l}\right)$ are known real matrices with appropriate dimensions. The frequency spectrum of the exogenous noise $u(i, j)$ is assumed to belong to a known rectangular region $\Omega$, where

$$
\begin{aligned}
\Omega \triangleq & \left\{\left(\mu_{1}, \mu_{2}\right) \in \mathbb{R} \mid \mu_{1}^{a} \leq \mu_{1} \leq \mu_{1}^{b} ; \mu_{2}^{a} \leq \mu_{2} \leq \mu_{2}^{b} ;\right. \\
& \left.\mu_{1}^{a}, \mu_{2}^{a}, \mu_{1}^{b}, \mu_{2}^{b} \in[-\pi, \pi]\right\}
\end{aligned}
$$

where $\mu_{1}^{a}, \mu_{1}^{b}, \mu_{2}^{a}$ and $\mu_{2}^{b}$ are known scalars.

A more compact presentation of the Takagi-Sugeno 2D discrete-time fuzzy model systems is given by

$$
\begin{aligned}
x_{i+1, j+1} & =A_{1}(h) x_{i, j+1}+A_{2}(h) x_{i+1, j} \\
& +B_{1}(h) u_{i, j+1}+B_{2}(h) u_{i+1, j} \\
y_{i, j} & =C(h) x_{i, j} \\
x(i, 0) & =x_{i, 0}, \quad x(0, j)=x_{0, j}, i, j \geq 0
\end{aligned}
$$


where

$$
\left[\begin{array}{cc}
A_{1}(h) & B_{1}(h) \\
A_{2}(h) & B_{2}(h) \\
C(h) &
\end{array}\right]=\sum_{l=1}^{r} h_{l}(\theta(k))\left[\begin{array}{cc}
A_{1 l} & B_{1 l} \\
A_{2 l} & B_{2 l} \\
C_{l} &
\end{array}\right]
$$

In this paper, we will approximate T-S fuzzy FM system (1) by the following reduced-order T-S model: Plant Rule l: IF $\theta_{1}(k)$ is $\tilde{N}_{1}^{l}, \theta_{2}(k)$ is $\tilde{N}_{2}^{l}, \ldots$ and $\theta_{\alpha}(k)$ is $\tilde{N}_{\alpha}^{l}$, Then,

$$
\begin{aligned}
\breve{x}_{i+1, j+1} & =\breve{A}_{1 l} \breve{x}_{i, j+1}+\breve{A}_{2 l} \breve{x}_{i+1, j}+\breve{B}_{1 l} u_{i, j+1}+\breve{B}_{2 l} u_{i+1, j} \\
\breve{y}_{i, j} & =\breve{C}_{l} \breve{x}_{i, j}
\end{aligned}
$$

where $\breve{x}_{i, j} \in \mathbb{R}^{\breve{n}}(\breve{n}<n)$ is the state vector; $\breve{y}_{i, j} \in \mathbb{R}^{y}$ is the output; and $\left(\breve{A}_{1 l}, \breve{A}_{2 l}, \breve{B}_{1 l}, \breve{B}_{2 l}, \breve{C}_{l}\right)$ are the parameters of reduced-order model that are appropriately dimensioned real matrices to be determined.

The reduced-order model can be written in a compact form

$$
\begin{aligned}
\breve{x}_{i+1, j+1} & =\breve{A}_{1}(h) \breve{x}_{i, j+1}+\breve{A}_{2}(h) x_{i+1, j} \\
& +\breve{B}_{1}(h) u_{i, j+1}+\breve{B}_{2}(h) u_{i+1, j} \\
\breve{y}_{i, j} & =\breve{C}(h) \breve{x}_{i, j}
\end{aligned}
$$

where

$$
\left[\begin{array}{cc}
\breve{A}_{1}(h) & \breve{B}_{1}(h) \\
\breve{A}_{2}(h) & \breve{B}_{2}(h) \\
\breve{C}(h) &
\end{array}\right]=\sum_{l=1}^{r} h_{l}(\theta(k))\left[\begin{array}{cc}
\breve{A}_{1 l} & \breve{B}_{1 l} \\
\breve{A}_{2 l} & \breve{B}_{2 l} \\
\breve{C}_{l} &
\end{array}\right]
$$

Defining the augmented state vector $\xi_{i, j}:=\left[\begin{array}{ll}x_{i, j}^{T} & \breve{x}_{i, j}^{T}\end{array}\right]^{T}$, $e_{i, j}=y_{i, j}-\breve{y}_{i, j}$, we can obtain the following approximation error system :

$$
\begin{aligned}
\xi_{i+1, j+1} & =\bar{A}_{1}(h) \xi_{i, j+1}+\bar{A}_{2}(h) \xi_{i+1, j} \\
& +\bar{B}_{1}(h) u_{i, j+1}+\bar{B}_{2}(h) u_{i+1, j} \\
e_{i, j} & =\bar{C}(h) \xi_{i, j}+\bar{D}(h) u_{i, j} \\
\bar{A}_{1}(h) & =\left[\begin{array}{cc}
A_{1}(h) & 0 \\
0 & \breve{A}_{1}(h)
\end{array}\right] ; \bar{B}_{1}(h)=\left[\begin{array}{l}
B_{1}(h) \\
\breve{B}_{1}(h)
\end{array}\right] ; \\
\mathcal{A}_{2}(h) & =\left[\begin{array}{cc}
A_{2}(h) & 0 \\
0 & \breve{A}_{2}(h)
\end{array}\right] ; \bar{B}_{2}(h)=\left[\begin{array}{l}
B_{2}(h) \\
\breve{B}_{2}(h)
\end{array}\right] ; \\
\bar{C}(h) & =\left[\begin{array}{cc}
C(h) & -\breve{C}(h)
\end{array}\right]
\end{aligned}
$$

Next, some related definitions for 2D T-S fuzzy FM model with FF ranges are given as follows:

The problem addressed in this work can be formulated as follows: Given TS fuzzy FM system (3), The objective is to design a suitable TS FM reduced-order model in the form of (6) such that the following two requirements are satisfied:

- Error system (8) is asymptotically stable when $u(i, j) \equiv 0$.

- Letting $\gamma>0$, be a given constant, under the zero-initial condition, equation

$$
\sup _{0 \neq u_{i, j} \in l_{2}\{[0, \infty),[0, \infty)\}}\left\|e_{i, j}\right\|_{2} \leq \gamma^{2}\left\|u_{i, j}\right\|_{2}
$$

holds for all solutions of (8) with $u_{i, j} \in$ $l_{2}\{[0, \infty),[0, \infty)\}$ such the following hold

$$
\begin{aligned}
& e^{j \mu_{3}^{b}} \sum_{i=0}^{\infty} \sum_{j=0}^{\infty}\left[\left(\xi_{i+1, j+1}-e^{j \mu_{1}^{a}} \xi_{i, j+1}\right)\right. \\
& \left.\left(\xi_{i+1, j+1}-e^{j \mu_{1}^{b}} \xi_{i, j+1}\right)^{T}\right] \leq 0 \\
& e^{j \mu_{4}^{b}} \sum_{i=0}^{\infty} \sum_{j=0}^{\infty}\left[\left(\xi_{i+1, j+1}-e^{j \mu_{2}^{a}} \xi_{i+1, j}\right)\right. \\
& \left.\left(\xi_{i+1, j+1}-e^{j \mu_{2}^{b}} \xi_{i+1, j}\right)^{T}\right] \leq 0
\end{aligned}
$$

where $\mu_{3}^{b}=\frac{\mu_{1}^{b}-\mu_{1}^{a}}{2} ; \quad \mu_{4}^{b}=\frac{\mu_{2}^{b}-\mu_{2}^{a}}{2}$

\subsection{Preliminaries}

We introduce the following technical lemmas that are useful for deriving our results.

Lemma 1 ([21]) From (13), we can obtain (14)

$$
\left[\begin{array}{cc}
T+M U+U^{T} M^{T} & * \\
-M^{T}+G U & V-G-G^{T}
\end{array}\right]<0
$$

$$
T+U^{T} V U<0
$$

Lemma 2 ([13]) Let $\gamma>0$ be a given scalar and a rectangular FF domain (2). For error system (8) is asymptotically stable, $H_{\infty}$ performance (11) is satisfied, if there exist Hermitian $P_{1}, P_{2}, 0<Q_{1}, 0<Q_{2} \in \mathbb{H}_{2 n}$, such that

$$
\begin{array}{r}
{\left[\begin{array}{cc}
\mathcal{A}(h) & \mathcal{B}(h) \\
I & 0
\end{array}\right]^{T}\left[\begin{array}{cc}
P & \Lambda^{*} Q \\
Q \Lambda & -\mathcal{R}
\end{array}\right]\left[\begin{array}{cc}
\mathcal{A}(h) & \mathcal{B}(h) \\
I & 0
\end{array}\right]} \\
+\left[\begin{array}{cc}
C(h)^{T} C(h) & 0 \\
0 & -\gamma^{2} I
\end{array}\right]<0
\end{array}
$$

where

$$
\begin{aligned}
\mathcal{A}(h) & =\left[\begin{array}{cc}
\bar{A}_{1}(h) & \bar{A}_{2}(h)
\end{array}\right] ; \mathcal{B}(h)=\left[\begin{array}{ll}
\bar{B}_{1}(h) & \bar{B}_{2}(h)
\end{array}\right] ; \\
\mathcal{C}(h) & =\left[\begin{array}{cc}
\bar{C}(h) & 0 \\
0 & \bar{C}(h)
\end{array}\right] ; \\
Q & =\left[\begin{array}{cc}
Q_{1} & Q_{2}
\end{array}\right] ; P=P_{1}+P_{2} ; \\
\Lambda & =\left[\begin{array}{cc}
e^{-j \mu_{3}^{a}} I_{2 \bar{n}} & 0 \\
0 & e^{-j \mu_{4}^{a}} I_{2 \bar{n}}
\end{array}\right] ; \\
\mathcal{R} & =\left[\begin{array}{cc}
P_{1}+2 \cos \left(\mu_{3}^{b}\right) Q_{1} & 0 \\
0 & P_{2}+2 \cos \left(\mu_{4}^{b}\right) Q_{2}
\end{array}\right] ; \\
\mu_{3}^{a} & =\frac{\mu_{1}^{a}+\mu_{1}^{b}}{2}, \quad \mu_{4}^{a}=\frac{\mu_{2}^{a}+\mu_{2}^{b}}{2} ; \\
\mu_{3}^{b} & =\frac{\mu_{1}^{b}-\mu_{1}^{a}}{2}, \quad \mu_{4}^{b}=\frac{\mu_{2}^{b}-\mu_{2}^{a}}{2}
\end{aligned}
$$

\section{Main results}

\section{1 $H_{\infty}$ Model Reduction analysis}

On the basis of Lemmas 1 and 2, we give the following theorem, which can guarantee the asymptotical stability and the $H_{\infty}$ performance of error system (8) in the FF domain of input noise. 
Theorem 1 Let $\gamma>0$ be a given scalar and a rectangular FF domain (2), a reduced-order model of form (6) exists such that the error system in (8) is asymptotically stable and $H_{\infty}$ performance (11) is satisfied, if and only if there exist Hermitian matrices $P=P_{1}+P_{2}, Q=\left[\begin{array}{ll}Q_{1} & Q_{2}\end{array}\right]$, symmetric matrices $W_{1}, W_{2}$ and matrices $M_{1}, M_{2}, G_{1}, G_{2}$, $F_{1}$ and $H$ satisfying $Q_{1}>0, Q_{2}>0, W_{1}>0, W_{2}>0$ and

$$
\begin{gathered}
\Phi=\left[\begin{array}{ccccc}
\Phi_{11} & \Phi_{12} & \Phi_{13} & \mathcal{A}^{T}(h) G_{2}^{T} & C^{T} \\
* & \Phi_{22} & \Phi_{23} & \mathcal{B}^{T}(h) G_{2}^{T} & \mathcal{D}^{T} \\
* & * & \Phi_{33} & \Lambda^{*} Q-G_{2}^{T} & 0 \\
* & * & * & -\mathcal{R} & 0 \\
* & * & * & * & -I
\end{array}\right]<0 \\
\Upsilon=\left[\begin{array}{ccc}
\Upsilon_{11} & F_{1} \bar{A}_{2}(h) & -F_{1}+\bar{A}_{1}^{T}(h)^{T} H^{T} \\
* & -W_{2} & \bar{A}_{2}^{T}(h)^{T} H^{T} \\
* & * & W_{1}+W_{2}-\operatorname{sym}(H)
\end{array}\right]<0
\end{gathered}
$$

$$
\begin{aligned}
\Phi_{11} & =M_{1} \mathcal{A}(h)+\mathcal{A}^{T}(h) M_{1}^{T} ; \\
\Phi_{12} & =M_{1} \mathcal{B}(h)+\mathcal{A}^{T}(h) M_{2}^{T} ; \\
\Phi_{13} & =\mathcal{A}^{T}(h) G_{1}^{T}-M_{1} ; \\
\Phi_{22} & =-\gamma^{2} I+M_{2} \mathcal{B}(h)+\mathcal{B}^{T}(h) M_{2}^{T} ; \\
\Phi_{23} & =\mathcal{B}^{T}(h) G_{1}^{T}-M_{2} ; \\
\Phi_{33} & =P-G_{1}-G_{1}^{T} ; \\
\Upsilon_{11} & =-W_{1}+\operatorname{sym}\left(F_{1} \bar{A}_{1}(h)\right)
\end{aligned}
$$

Proof 1 First, we prove that (15) is equivalent to (17). Condition (15) can be rewritten as

$$
T+U^{T} V U<0
$$

Where

$$
\begin{aligned}
U & =\left[\begin{array}{cc}
\mathcal{A}(h) & \mathcal{B}(h) \\
I & 0
\end{array}\right] ; \quad V=\left[\begin{array}{cc}
P & \Lambda^{*} Q \\
Q \Lambda & -\mathcal{R}
\end{array}\right] ; \\
T & =\left[\begin{array}{cc}
C(h)^{T} \mathcal{C}(h) & C(h)^{T} \mathcal{D}(h) \\
\mathcal{D}(h)^{T} \mathcal{C}(h) & -\gamma^{2} I+\mathcal{D}(h)^{T} \mathcal{D}(h)
\end{array}\right]
\end{aligned}
$$

At this stand, by using Lemma 2, (19) is equivalent to

$$
\left[\begin{array}{cc}
T+M U+U^{T} M^{T} & U^{T} G^{T}-M \\
-M^{T}+G U & V-G-G^{T}
\end{array}\right]<0
$$

We chose $M$ and $G$ are expressed as the following structures:

$$
M=\left[\begin{array}{cc}
M_{1} & 0 \\
M_{2} & 0
\end{array}\right] ; \quad G=\left[\begin{array}{cc}
G_{1} & 0 \\
G_{2} & 0
\end{array}\right]
$$

which, using Schur complement, leads to given (17).

Second step, let us construct a Lyapunov function inequality, $\bar{A}_{1}(h)$ and $\bar{A}_{2}(h)$ is stable if and only if there exist symmetric matrices $W_{1}>0, W_{2}>0$ such that

$$
\begin{aligned}
& {\left[\begin{array}{cc}
\bar{A}_{1}(h) & \bar{A}_{2}(h)
\end{array}\right]^{T}\left(W_{1}+W_{2}\right)\left[\begin{array}{cc}
\bar{A}_{1}(h) & \bar{A}_{2}(h)
\end{array}\right] } \\
- & {\left[\begin{array}{cc}
W_{1} & 0 \\
0 & W_{2}
\end{array}\right]<0 }
\end{aligned}
$$

which is rewritten in the form

$$
\hat{T}+\hat{U}^{T} \hat{V} \hat{U}<0
$$

Where

$$
\begin{aligned}
\hat{T} & =\left[\begin{array}{cc}
-W_{1} & 0 \\
0 & -W_{2}
\end{array}\right] ; \hat{V}=W_{1}+W_{2} ; \\
\hat{U} & =\mathcal{A}(h)=\left[\begin{array}{ll}
\bar{A}_{1}(h) & \bar{A}_{2}(h)
\end{array}\right] ;
\end{aligned}
$$

We chose F follows:

$$
F=\left[\begin{array}{ll}
F_{1} & 0
\end{array}\right]^{T}
$$

Using Lemma 1, (24-25) are equivalent to (18).

\subsection{Finite Frequency $H_{\infty}$ Model Reduction design}

The main objective is to determine the reduced-order matrices such that error system (8) is asymptotically stable and guarantees an $H_{\infty}$ disturbance attenuation level $\gamma$ and satisfies the FF in (11).

Theorem 2 Let $\gamma>0$ be a given scalar and a rectangular FF domain (2), a reduced-order model of form (6) exists such that the error system in (8) is asymptotically stable and $H_{\infty}$ performance (11) is satisfied, if there exist matrices $\hat{A}_{1 i}, \hat{B}_{1 i}, \hat{A}_{2 i}, \hat{B}_{2 i}, \hat{C}_{1 i}, \hat{D}_{1 i}, M_{1 u}, G_{2 u}, M_{2 t}, G_{1 t}, H_{1 t}, F_{1 t}$, $V, u=1,2,3,4, t=1,2, P_{1 s}, Q_{1 s}>0, W_{1 s}>0, P_{2 s}$, $Q_{2 s}>0, W_{2 s}>0, s=1,2,3$, satisfying

$$
\tilde{\Psi}=\left[\begin{array}{c|c}
\tilde{\Psi}_{1} & \tilde{\Psi}_{2} \\
\hline * & \tilde{\Psi}_{3}
\end{array}\right]<0
$$

$$
\tilde{\Upsilon}=\left[\begin{array}{cccccc}
\tilde{\Upsilon}_{11} & \tilde{\Upsilon}_{12} & \tilde{\Upsilon}_{13} & E \hat{A}_{2 i} & \tilde{\Upsilon}_{15} & \tilde{\Upsilon}_{16} \\
* & \tilde{\Upsilon}_{22} & \tilde{\Upsilon}_{23} & \hat{A}_{2 i}^{T} & \tilde{\Upsilon}_{25} & \tilde{\Upsilon}_{26} \\
* & * & -W_{21} & -W_{22} & \tilde{\Upsilon}_{35} & A_{2 H}^{T} H_{12}^{T} \\
* & * & * & -W_{23} & \tilde{\Upsilon}_{45} & \hat{A}_{2 i}^{T} \\
* & * & * & * & \tilde{\Upsilon}_{55} & \tilde{\Upsilon}_{56}^{T} \\
* & * & * & * & * & \tilde{\Upsilon}_{66}
\end{array}\right]<0
$$

$$
\tilde{\Psi}_{1}=\left[\begin{array}{cccccc}
\tilde{\Psi}_{111} & \tilde{\Psi}_{112} & \tilde{\Psi}_{113} & \tilde{\Psi}_{14} & \tilde{\Psi}_{115} & \tilde{\Psi}_{116} \\
* & \tilde{\Psi}_{122} & M_{12} A_{2 j} & s y m\left[\hat{A}_{2 i}\right] & \tilde{\Psi}_{125} & \tilde{\Psi}_{126} \\
* & * & \tilde{\Psi}_{133} & A_{2 j}^{T} M_{14}^{T} & \tilde{\Psi}_{135} & \tilde{\Psi}_{136} \\
* & * & * & \tilde{\Psi}_{144} & \tilde{\Psi}_{145} & \tilde{\Psi}_{146} \\
* & * & * & * & \tilde{\Psi}_{155} & \tilde{\Psi}_{156} \\
* & * & * & * & * & \tilde{\Psi}_{166}
\end{array}\right] ;
$$

$$
\begin{aligned}
\tilde{\Psi}_{2} & =\left[\begin{array}{cccccccc}
\tilde{\Psi}_{11} & \tilde{\Psi}_{12} & \tilde{\Psi}_{13} & \tilde{\Psi}_{14} & \tilde{\Psi}_{15} & \tilde{\Psi}_{16} & \tilde{\Psi}_{17} & 0 \\
\tilde{\Psi}_{21} & \tilde{\Psi}_{22} & \tilde{\Psi}_{23} & \tilde{\Psi}_{24} & 0 & \tilde{\Psi}_{26} & 0 & \tilde{\Psi}_{58} \\
\tilde{\Psi}_{31} & \tilde{\Psi}_{32} & \tilde{\Psi}_{33} & \tilde{\Psi}_{34} & \tilde{\Psi}_{35} & \tilde{\Psi}_{36} & \tilde{\Psi}_{37} & 0 \\
\tilde{\Psi}_{41} & \tilde{\Psi}_{42} & \tilde{\Psi}_{43} & \tilde{\Psi}_{44} & 0 & \tilde{\Psi}_{46} & 0 & -\hat{C}_{i}^{T} \\
\tilde{\Psi}_{51} & \tilde{\Psi}_{52} & \tilde{\Psi}_{53} & \tilde{\Psi}_{54} & \tilde{\Psi}_{55} & \tilde{\Psi}_{56} & \tilde{\Psi}_{57} & 0 \\
\tilde{\Psi}_{61} & \tilde{\Psi}_{62} & \tilde{\Psi}_{63} & \tilde{\Psi}_{64} & \tilde{\Psi}_{65} & \tilde{\Psi}_{66} & 0 & 0
\end{array}\right] ; \\
\tilde{\Psi}_{3} & =\left[\begin{array}{cccccccc}
\tilde{\Psi}_{31} & \tilde{\Psi}_{312} & \tilde{\Psi}_{313} & \tilde{\Psi}_{314} & \tilde{\Psi}_{315} & \tilde{\Psi}_{316} & 0 & 0 \\
* & \tilde{\Psi}_{322} & \tilde{\Psi}_{33} & \tilde{\Psi}_{34} & \tilde{\Psi}_{325} & \tilde{\Psi}_{326} & 0 & 0 \\
* & * & \tilde{\Psi}_{333} & \tilde{\Psi}_{34} & 0 & 0 & 0 & 0 \\
* & * & * & \tilde{\Psi}_{344} & 0 & 0 & 0 & 0 \\
* & * & * & * & \tilde{\Psi}_{355} & \tilde{\Psi}_{356} & 0 & 0 \\
* & * & * & * & * & \tilde{\Psi}_{366} & 0 & 0 \\
* & * & * & * & * & * & -I & 0 \\
* & * & * & * & * & * & * & -I
\end{array}\right]
\end{aligned}
$$




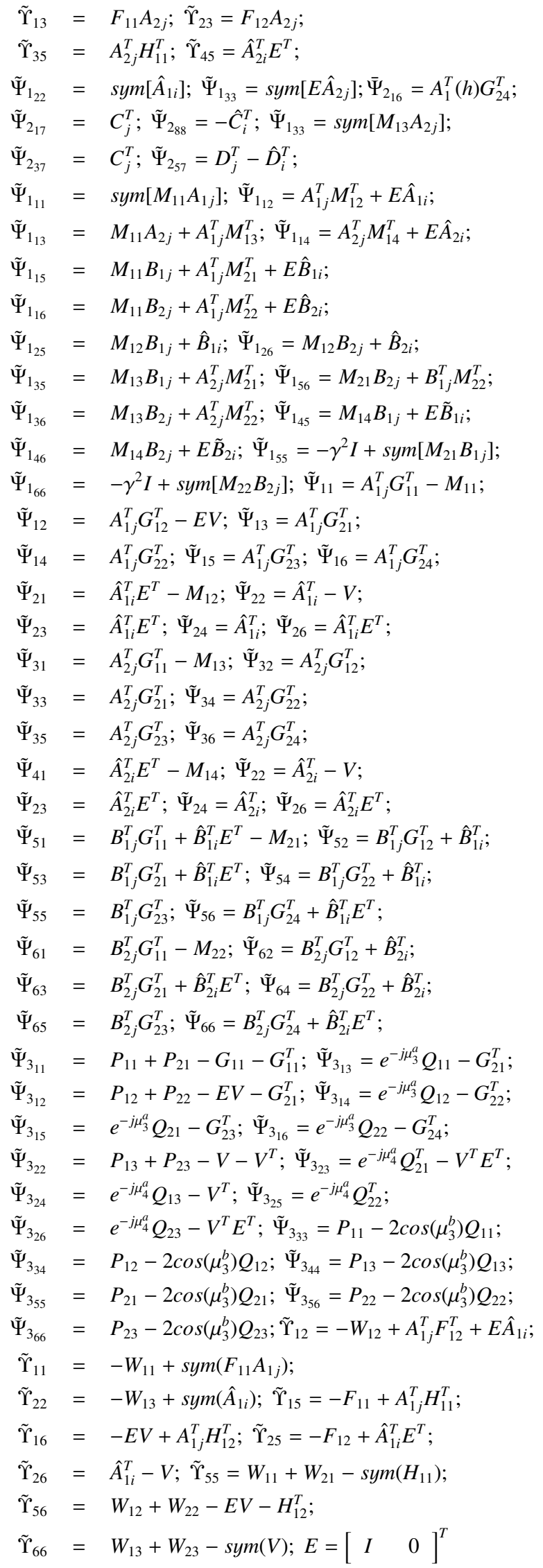

The reduced-order parameters are given by:
Proof 2 Parameterise slack matrices $M_{1}, M_{2}, G_{1}, G_{2}, F_{1}$ and $H$ in Theorem 1 as

$$
\begin{aligned}
M_{1} & =\left[\begin{array}{cc}
M_{11} & E V \\
M_{12} & V \\
M_{13} & 0 \\
M_{14} & E V
\end{array}\right] ; G_{2}=\left[\begin{array}{cc}
G_{21} & E V \\
G_{22} & V \\
G_{23} & 0 \\
G_{24} & E V
\end{array}\right] ; \\
M_{2} & =\left[\begin{array}{cc}
M_{21} & 0 \\
M_{22} & 0
\end{array}\right] ; G_{1}=\left[\begin{array}{cc}
G_{11} & E V \\
G_{12} & V
\end{array}\right] ; \\
F & =\left[\begin{array}{cc}
F_{11} & E V \\
F_{12} & V
\end{array}\right], H=\left[\begin{array}{cc}
H_{11} & E V \\
H_{12} & V
\end{array}\right]
\end{aligned}
$$

Moreover, for matrix variables $P_{1}, Q_{1}>0, P_{2}$, $Q_{2}>0, W_{1}>0, W_{2}>0$ in theorem 1 , we define:

$$
\begin{aligned}
P_{1} & =\left[\begin{array}{cc}
P_{11} & P_{12} \\
* & P_{13}
\end{array}\right] ; P_{2}=\left[\begin{array}{cc}
P_{21} & P_{22} \\
* & P_{23}
\end{array}\right] ; \\
Q_{1} & =\left[\begin{array}{cc}
Q_{11} & Q_{12} \\
* & Q_{13}
\end{array}\right] ; Q_{2}=\left[\begin{array}{cc}
Q_{21} & Q_{22} \\
* & Q_{23}
\end{array}\right] ; \\
W_{1} & =\left[\begin{array}{cc}
W_{11} & W_{12} \\
* & W_{13}
\end{array}\right] ; W_{2}=\left[\begin{array}{cc}
W_{21} & W_{22} \\
* & W_{23}
\end{array}\right]
\end{aligned}
$$

In addition, by replacing (9), (16) and into (17) and (18), and combining (30), (32), we obtain theorem 1, where

$$
\begin{aligned}
& \hat{A}_{1}(h)=V \breve{A}_{1}(h), \hat{A}_{2}(h)=V \breve{A}_{2}(h), \\
& \hat{B}_{1}(h)=V \breve{B}_{1}(h), \hat{B}_{2}(h)=V \breve{B}_{2}(h),
\end{aligned}
$$

Remark 1 If we take $Q_{s}=\operatorname{diag}\left\{Q_{s h}, Q_{s v}\right\}=0, s=1,2,3$, we can use Theorem 2 to solve the $H_{\infty}$ model reduction problem in the entire frequency $(E F)$ domain of twodimensional (2D) T-S fuzzy discrete systems described by FMLSS model.

\section{Numerical Example}

Considering a 2D discrete-time T-S fuzzy system with two rules, whose matrices are given as [19] Plant Rule 1: IF $\theta_{1}(k)$ is $\tilde{N}_{1}^{1}, \theta_{2}(k)$ is $\tilde{N}_{2}^{1}$, Then,

$$
\begin{aligned}
x_{i+1, j+1} & =A_{11} x_{i, j+1}+A_{21} x_{i+1, j}+B_{11} u_{i, j+1}+B_{21} u_{i+1, j} \\
y_{i, j} & =C_{1} x_{i, j}
\end{aligned}
$$

Plant Rule 2: IF $\theta_{1}(k)$ is $\tilde{N}_{1}^{2}, \theta_{2}(k)$ is $\tilde{N}_{2}^{2}$, Then,

$$
\begin{aligned}
x_{i+1, j+1} & =A_{12} x_{i, j+1}+A_{22} x_{i+1, j}+B_{12} u_{i, j+1}+B_{22} u_{i+1, j} \\
y_{i, j} & =C_{2} x_{i, j}
\end{aligned}
$$


where

$$
\begin{aligned}
& A_{11}=A_{12}=\left[\begin{array}{cccc}
0 & 0 & 0 & 0 \\
0 & 0 & 0 & 0 \\
-0.05 & 0 & 1.05 & 0 \\
0 & 0.05 & 0 & 0.95
\end{array}\right] \text {; } \\
& A_{21}=A_{22}=\left[\begin{array}{llll}
0 & 0 & 1 & 0 \\
0 & 0 & 0 & 0 \\
0 & 0 & 0 & 0 \\
0 & 0 & 0 & 0
\end{array}\right] B_{11}=\left[\begin{array}{c}
-0.43 \\
0 \\
0.05 \\
0
\end{array}\right] \text {; } \\
& B_{12}=\left[\begin{array}{c}
-0.03 \\
0 \\
0 \\
-0.04
\end{array}\right] ; B_{21}=\left[\begin{array}{c}
-0.04 \\
0 \\
0.01 \\
-0.02
\end{array}\right] ; B_{22}=\left[\begin{array}{c}
0 \\
-0.01 \\
0.01 \\
0
\end{array}\right] \text {; } \\
& C_{1}=\left[\begin{array}{cccc}
-0.03 & 0 & -0.02 & -0.03 \\
0.02 & -0.04 & 0 & -0.04 \\
-0.04 & 0 & -0.01 & 0.02 \\
0 & 0 & -0.02 & 0.02
\end{array}\right] \text {; } \\
& C_{1}=\left[\begin{array}{cccc}
-0.02 & -0.04 & 0.02 & 0 \\
0.01 & -0.03 & -0.05 & 0 \\
-0.02 & 0 & 0 & 0 \\
-0.05 & 0 & 0 & -0.03
\end{array}\right] \text {; } \\
& D_{1}=\left[\begin{array}{c}
-0.03 \\
-0.02 \\
-0.05 \\
0.03
\end{array}\right] \text {. }
\end{aligned}
$$

The normalized membership function : where

$$
h_{1}\left(\theta_{i, j}\right)=\frac{\sin ^{2}\left(x_{1}(i, j)\right)}{2+\cos ^{2}\left(x_{1}(i, j)\right)} ; h_{2}\left(\theta_{i, j}\right)=1-\frac{\sin ^{2}\left(x_{1}(i, j)\right)}{2+\cos ^{2}\left(x_{1}(i, j)\right)}
$$

To demonstrate the value of our proposed approach, we provide in Table 1 the $H_{\infty}$ reduced order performance levels, which shows the conservativeness of the FF method.

\begin{tabular}{l|c|c}
\hline Frequency & Methods & $\gamma$ \\
\hline$[0, \pi] \times[0, \pi]$ & Theorem 2 $(\mathbf{Q}=\mathbf{0})$ & $\mathbf{0 . 8 2 4 5}$ \\
\hline$\left[\frac{\pi}{8}, \frac{\pi}{3}\right] \times\left[\frac{\pi}{8}, \frac{\pi}{3}\right]$ & Theorem 2 & $\mathbf{0 . 2 8 4 5}$ \\
\hline
\end{tabular}

Table 1: Comparison of reduced-order performance obtained in different ranges.

Assume that the reduced-order model in (8) has an order FM LSS model $\breve{n}=2$, for FF domain $\left[\frac{\pi}{8}, \frac{\pi}{3}\right] \times\left[\frac{\pi}{8}, \frac{\pi}{3}\right]$, the reduced-order parameters are given as follows:

$$
\begin{aligned}
& \breve{A}_{11}=\left[\begin{array}{cc}
-0.1921 & 0.0245 \\
-0.8514 & -0.8845
\end{array}\right] ; \breve{B}_{11}=\left[\begin{array}{c}
-0.3219 \\
0.0002
\end{array}\right] ; \\
& \breve{A}_{12}=\left[\begin{array}{cc}
-0.1605 & 0.0378 \\
-0.6405 & -0.8715
\end{array}\right] ; \breve{B}_{21}=\left[\begin{array}{c}
-0.0923 \\
-0.1105
\end{array}\right] ; \\
& \breve{A}_{21}=\left[\begin{array}{cc}
-0.1527 & 0.7145 \\
0.0305 & -0.2105
\end{array}\right] ; \breve{B}_{12}=\left[\begin{array}{c}
-0.1504 \\
0.0204
\end{array}\right] ; \\
& \breve{C}_{1}=\left[\begin{array}{cc}
-0.0978 & 0.0345 \\
0.0374 & -0.0547 \\
0.0125 & -0.2147 \\
-0.0248 & 0.2345
\end{array}\right] ; \breve{B}_{22}=\left[\begin{array}{c}
-0.0017 \\
0.0201
\end{array}\right] ; \\
& \breve{C}_{2}=\left[\begin{array}{cc}
-0.1106 & -0.1503 \\
0.0748 & 0.1875 \\
0.1100 & 0.0147 \\
0.0101 & -0.2974
\end{array}\right] ; \\
& \breve{A}_{22}=\left[\begin{array}{cc}
-0.0101 & 0.8745 \\
0.0174 & -0.0202
\end{array}\right] .
\end{aligned}
$$

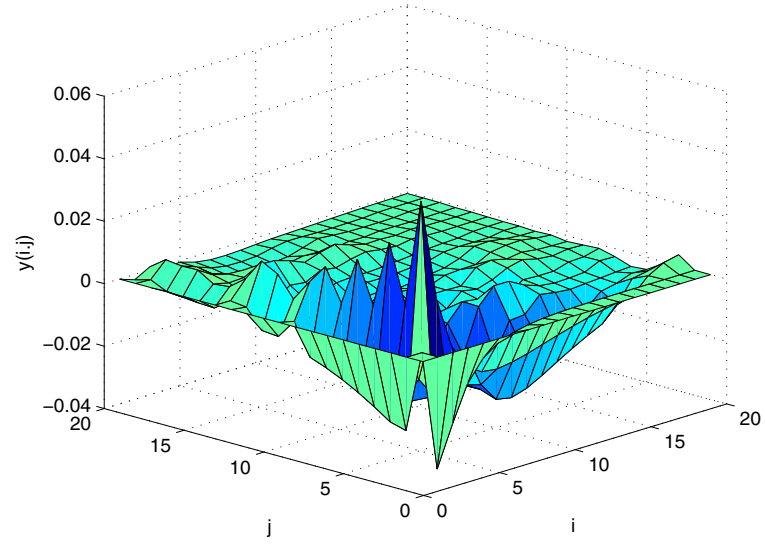

Figure 1. Output response $y_{i, j}$ of fuzzy systems in (3).

Furthermore, under zero boundary conditions, by calculation, we have $\frac{\left\|e_{i, j}\right\|_{2}}{\left\|u_{i, j}\right\|_{2}}=0.2715$, which is below the corresponding prescribed value $\gamma=0.2845$, showing the effectiveness of the model reduction design method.

The trajectories of $y_{i, j}, \breve{y}_{i, j}$ and the error system $e_{i, j}$ are shown in Figures 2, 3 and 4. It is clear that effectively, the $2 \mathrm{D}$ system is asymptotically stable and converges towards zero. All the simulation results show the effectiveness of the designed reduced-order system.

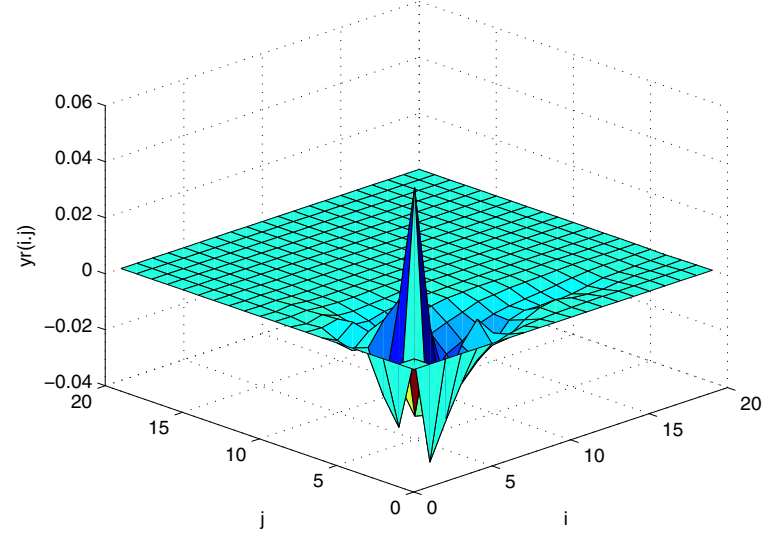

Figure 2. Output response $\breve{y}_{i, j}$ of reduced-order system in (6).

\section{Conclusion}

This paper has investigated the FF $H_{\infty}$ reduced model design problem for two-dimensional (2D) discrete-time T-S fuzzy systems described by FMLSS model. By applying gKYP lemma for 2D discrete systems, we introduce many slack matrices to provide extra free dimensions in the so(36) lution space of the $H_{\infty}$ optimization. 


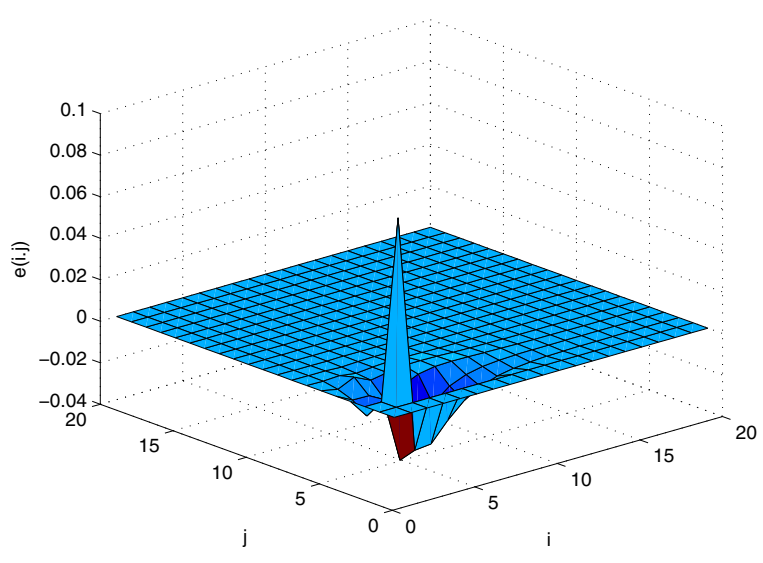

Figure 3. Error response of $e_{i, j}$.

\section{References}

[1] T. Kaczorek, Ed. Two-Dimensional Linear Systems. Lecture Notes in Control and Information Science, New York: Springer-Verlag, 68, 1985.

[2] C. Du and L. Xie. $H_{\infty}$ Control and Filtering of Twodimensional Systems. Springer Verlag, 278, 2002.

[3] X. Li, J. Lam, H. Gao, Y. Gu, A frequencypartitioning approach to stability analysis of twodimensional discrete systems. Multidimensional Systems and Signal Processing, 26(1):67-93, 2013.

[6] H. Gao, J. Lam, C. Wang, \& S. Xu. $H_{\infty}$ model reduction for uncertain two-dimensional discrete systems. Optimal Control Applications and Methods, 26(4):199227, 2005.

[7] T. Takagi \& M. Sugeno. Fuzzy identification of systems and its applications to modeling and control. IEEE Transactions on Systems, Man, and Cybernetics, 15(1) : 116-132, 1985.

[9] K. Glover. All optimal Hankel-norm approximations of linear multivariable systems and their $L^{\infty}$ error bounds. International journal of control, 39(6) : 1115-1195, 1984.

[10] G. J. Lastman. Reduced-order aggregated models for bilinear time invariant dynamical systems. IEEE Transactions on Automatic Control, 29: 359-361, 1984.

[11] A. Ghafoor, J. Wang, and V. Sreeram. Frequencyweighted model reduction method with error bounds for 2-D separable denominator discrete systems. IEEE International Symposium on, Mediterrean Conference on Control and Automation Intelligent Control, 525530. 2005.
[12] Z. Duan, J. Shen, I. Ghous, \& J. Fu. $H_{\infty}$ filtering for discrete-time 2D T-S fuzzy systems with finite frequency disturbances. IET Control Theory and Applications, 13(13) : 1983-1994, 2019.

[13] Z.Duan, J. Zhou, \& J. Shen. Filter design for discrete-time two-dimensional T-S fuzzy systems with finite frequency specification. International Journal of Systems Science, 50(3) : 599-613, 2019.

[14] H. Gao, X. Meng, T. Chen. New Design of Robust $H_{\infty}$ Filters for 2-D Systems. IEEE Signal Processing Letters, 15217-220, 2008.

[15] X. Li, H. Gao. Robust finite frequency image filtering for uncertain 2-D systems: The FM model case. Automatica, 29(8) : 2446-2452, 2013.

[16] Z.Duan, J. Zhou, \& J. Shen. Finite frequency filter design for nonlinear 2-D continuous systems in T-S form. Journal of the Franklin Institute, 354(18) : 86068625, 2017.

[17] X. Li, J. Lam, \& K. C. Cheung. Generalized $H_{\infty}$ model reduction for stable two-dimensional discrete systems. Multidimensional Systems and Signal Processing, vol. 27(2) : 359-382, 2016.

[18] A. El-Amrani, B. Boukili, A. Hmamed, A. El Hajjaji $\&$ I. Boumhidi. Robust $H_{\infty}$ filtering for 2D continuous systems with finite frequency specifications. International Journal of Systems Science, 49(1) : 43-57, 2017.

[19] Luo, Y., Wang, Z., Wei, G., \& Alsaadi, F. E. Robust $H_{\infty}$ Filtering for a Class of Two-Dimensional Uncertain Fuzzy Systems With Randomly Occurring Mixed Delays. IEEE Transactions on Fuzzy Systems, 25(1), 70-83. 2016.

[20] T. Hinamoto. Stability of 2-D discrete systems described by the Fornasini-Marchesini second model. IEEE Transactions on Circuits and Systems I: Fundamental Theory and Applications, 44(3): 254-257, 1997.

[21] D. Peaucelle. Unified Formulation for Robust Analysis and Synthesis with Parameter Dependent Lyapunov Functions. doctoral thesis of Toulouse III University, France, 2000.

[22] H. Tuan, P. Apkarian, T. Nguyen, T. Narikiyo, Robust mixed $\mathrm{H}_{2}-H_{\infty}$ filtering of 2D systems. IEEE Trans. Signal Process. 50(7), 1759-1771, 2002.

[23] Wu, L., Z. Wang, H. Gao, and C. Wang, $H_{\infty}$ and $L_{2}-L_{\infty}$ filtering for two-dimensional linear parametervarying systems. Int. J. Robust Nonlinear Control, Vol. 17(12), 1129-1154, 2007.

[25] X. Liu, and P. Shi. Robust $H_{\infty}$ filtering for 2-D systems with intermittent measurements Circuits, Systems and Signal Processing, Vol. 28(2), 283-303, 2009. 